\title{
Synthesis and antibacterial activity of new aryl / alkyl phosphonates via Michaelis-Arbuzov rearrangement
}

\author{
Gandavaram Syam Prasad, ${ }^{a}$ Manubolu Manjunath, ${ }^{b}$ Kachi Reddy Kishore Kumar Reddy, \\ Obulam Vijaya Sarathi Reddy, ${ }^{\text {b }}$ and Cirandur Suresh Reddy*,a \\ ${ }^{a}$ Department of Chemistry $/{ }^{b}$ Biochemistry, Sri Venkateswara University, Tirupati-517 502, India, \\ E-mail: csureshsvu@yahoo.com
}

\begin{abstract}
Synthesis of new aryl / alkyl phosphonates 3a-j has been accomplished via a MichaelisArbuzov-type rearrangement by the reaction of aryl / alkyl halide (1a-j) with triethyl phosphite (2) in dry toluene at reflux temperature. Products 3a-j were characterized by IR, ${ }^{13} \mathrm{C}$ and ${ }^{31} \mathrm{P}$ NMR and their antibacterial activity was evaluated.
\end{abstract}

Keywords: Aryl / alkyl phosphonates, antibacterial

\section{Introduction}

Phosphorus compounds containing the P-C bond are not particularly abundant in nature. Their diverse biological activity, ${ }^{1,2}$ has for a long time attracted considerable synthetic ${ }^{3}$ and pharmacological interest. ${ }^{4}$ The Michaelis-Arbuzov rearrangement, also known as the Arbuzov reaction, is very versatile way to form P-C bond from the reaction of an aryl / alkyl halide and trialkyl phosphite. ${ }^{5}$ This reaction is one of the most extensively investigated and is widely used to prepare phosphonates, phosphinates and phosphine oxides. ${ }^{6}$ Michaelis-Arbuzov reaction on the solid surface assisted by microwave heating for synthesis of organophsophorus compounds ${ }^{7-9}$ and also for the phosphonylation of aromatic compounds has been realized under the catalytical conditions. ${ }^{10-12}$ Without catalyst, Michaelis-Arbuzov rearrangement can only be carried out with highly activated benzene compounds by heating them with phosphites to yield the corresponding phosphonates. ${ }^{13}$ In our work we synthesized aryl / alkyl phosphonates without catalyst and under the mild conditions. 


\section{Results and Discussion}

The synthetic route involves reaction of aryl / alkyl halides (1a-j) with triethyl phosphite (2) in toluene at reflux temperature (Scheme 1) and the formation of new aryl / alkyl phosphonates 3a-j involved Michaelis-Arbuzov rearrangement. The chemical structures of all the new compounds were confirmed by elemental analysis, $\mathrm{IR}^{14,15}$ (Table 1), ${ }^{1} \mathrm{H}^{\mathrm{NMR}}{ }^{13}$ (Table 1) and ${ }^{13} \mathrm{C}$ NMR (Table 2) and ${ }^{31} \mathrm{P} \mathrm{NMR}^{16 a}$ (Table 2) spectral data.

Table 1. Physical and IR spectral data of 3a-j

\begin{tabular}{|c|c|c|c|c|c|c|c|}
\hline \multirow[t]{2}{*}{ Compd. ${ }^{a}$} & \multirow[t]{2}{*}{ Yield $^{\mathrm{b}}$} & \multirow{2}{*}{$\begin{array}{l}\text { Mol. Formula } \\
\text { (Mol. Wt) }\end{array}$} & \multicolumn{3}{|c|}{$\begin{array}{l}\text { Elemental analysis (\%) found } \\
\text { (Calcd.) }\end{array}$} & \multicolumn{2}{|c|}{$\operatorname{IR}\left(\mathrm{cm}^{-1}\right)$} \\
\hline & & & $\mathrm{C}$ & $\mathrm{H}$ & $\mathrm{N}$ & $\mathrm{P}=\mathrm{O}$ & $\mathrm{P}-\mathrm{C}$ \\
\hline $3 \mathbf{a}$ & 68 & $\begin{array}{l}\mathrm{C}_{10} \mathrm{H}_{15} \mathrm{O}_{4} \mathrm{P} \\
(230.20)\end{array}$ & $\begin{array}{l}52.12 \\
(52.18\end{array}$ & $\begin{array}{l}6.47 \\
6.57)\end{array}$ & - & 1211 & 981 \\
\hline $3 b$ & 84 & $\begin{array}{c}\mathrm{C}_{10} \mathrm{H}_{13} \mathrm{~N}_{2} \mathrm{O}_{7} \mathrm{P} \\
(304.19)\end{array}$ & $\begin{array}{l}39.37 \\
(39.48\end{array}$ & $\begin{array}{l}4.27 \\
4.31\end{array}$ & $\begin{array}{l}9.09 \\
9.21)\end{array}$ & 1240 & 960 \\
\hline $3 c$ & 68 & $\begin{array}{l}\mathrm{C}_{6} \mathrm{H}_{13} \mathrm{O}_{5} \mathrm{P} \\
(196.14)\end{array}$ & $\begin{array}{l}36.62 \\
(36.74\end{array}$ & $\begin{array}{l}6.49 \\
6.68)\end{array}$ & - & 1229 & 991 \\
\hline 3d & 65 & $\begin{array}{l}\mathrm{C}_{11} \mathrm{H}_{15} \mathrm{O}_{5} \mathrm{P} \\
(258.21)\end{array}$ & $\begin{array}{l}50.97 \\
(51.17\end{array}$ & $\begin{array}{l}5.72 \\
5.86)\end{array}$ & - & 1209 & 1021 \\
\hline $3 e$ & 63 & $\begin{array}{l}\mathrm{C}_{11} \mathrm{H}_{15} \mathrm{O}_{5} \mathrm{P} \\
(258.21)\end{array}$ & $\begin{array}{l}51.03 \\
(51.17\end{array}$ & $\begin{array}{l}5.78 \\
5.86)\end{array}$ & - & 1215 & 1015 \\
\hline $3 f$ & 82 & $\begin{array}{c}\mathrm{C}_{10} \mathrm{H}_{14} \mathrm{NO}_{5} \mathrm{P} \\
(259.20)\end{array}$ & $\begin{array}{l}46.14 \\
(46.34\end{array}$ & $\begin{array}{l}5.29 \\
5.44\end{array}$ & $\begin{array}{l}5.24 \\
5.40)\end{array}$ & 1245 & 1014 \\
\hline $3 g$ & 62 & $\begin{array}{l}\mathrm{C}_{11} \mathrm{H}_{15} \mathrm{O}_{4} \mathrm{P} \\
(242.21)\end{array}$ & $\begin{array}{l}54.37 \\
(54.55\end{array}$ & $\begin{array}{l}6.10 \\
6.24)\end{array}$ & - & 1232 & 1015 \\
\hline $3 \mathrm{~h}$ & 60 & $\begin{array}{l}\mathrm{C}_{11} \mathrm{H}_{17} \mathrm{O}_{4} \mathrm{P} \\
(244.22)\end{array}$ & $\begin{array}{l}53.96 \\
(54.10\end{array}$ & $\begin{array}{l}6.85 \\
7.02)\end{array}$ & - & 1237 & 1009 \\
\hline $3 \mathbf{i}$ & 64 & $\begin{array}{l}\mathrm{C}_{11} \mathrm{H}_{17} \mathrm{O}_{4} \mathrm{P} \\
(244.22)\end{array}$ & $\begin{array}{l}53.92 \\
(54.10\end{array}$ & $\begin{array}{l}6.86 \\
7.02)\end{array}$ & - & 1232 & 967 \\
\hline $\mathbf{3 j}$ & 67 & $\begin{array}{c}\mathrm{C}_{7} \mathrm{H}_{16} \mathrm{NO}_{7} \mathrm{P} \\
(257.18) \\
\end{array}$ & $\begin{array}{l}32.55 \\
(32.69 \\
\end{array}$ & $\begin{array}{l}6.09 \\
6.27 \\
\end{array}$ & $\begin{array}{l}5.26 \\
5.45)\end{array}$ & 1248 & 960 \\
\hline
\end{tabular}

${ }^{a}$ Obtained viscous liquids that decompose on attempted vacuum distillation.

${ }^{\mathrm{b}}$ After separation from the column chromatography.

The ${ }^{31} \mathrm{P}$ NMR spectral data for $\mathbf{3 a}-\mathbf{j}$ are given in the Table 2 . The ${ }^{31} \mathrm{P}$ NMR signals for $\mathbf{3 b}, \mathbf{3} \mathbf{c}$, 3e and 3g-i appeared as two distinct signals in the range of -1.28 to -2.08 and 5.74 to $20.71 \mathrm{ppm}$. This may be due to the presence of two isomers ${ }^{16 \mathrm{~b}, 16 \mathrm{c}}$ in the solution state with sufficient internal energy difference and considerable stability that enable measurement of their ${ }^{31} \mathrm{P} N M R$ resonance. The other compounds $\mathbf{3 a}, \mathbf{3 d}, \mathbf{3} \mathbf{f}$ and $\mathbf{3 j}$ gave only one ${ }^{31} \mathrm{P}$ NMR signal in the range of -1.29 to -1.50 and $7.09 \mathrm{ppm}$. 
Table 2. $\left({ }^{1} \mathrm{H},{ }^{13} \mathrm{C}\right.$ and $\left.{ }^{31} \mathrm{P}\right)$ NMR spectral data $\left(\delta, \mathrm{CDCl}_{3}\right)$ of $\mathbf{3 a - j}$

\begin{tabular}{|c|c|c|c|}
\hline Compd. & ${ }^{1} \mathrm{H}$ NMR & ${ }^{13} \mathrm{C}$ NMR & ${ }^{31} \mathrm{P}$ NMR \\
\hline 3a & $\begin{array}{c}\mathrm{Ar}-\mathrm{H}: 7.69(\mathrm{~d}=7.4 \mathrm{~Hz}, 2 \mathrm{H}) \\
6.75(\mathrm{~d}=7.8 \mathrm{~Hz}, 2 \mathrm{H}) \\
\mathrm{OCH}_{2}: 3.71-4.16(\mathrm{~m}, 4 \mathrm{H}) \\
\mathrm{CH}_{3}: 1.21-1.35(\mathrm{~m}, 6 \mathrm{H})\end{array}$ & $\begin{array}{c}155.54,132.14,117.28,111.82 \\
62.49,16.02\end{array}$ & 7.09 \\
\hline $3 b$ & $\begin{array}{l}\mathrm{Ar}-\mathrm{H}: 7.31-8.68(\mathrm{~m}, 6 \mathrm{H}) \\
\mathrm{OCH}_{2}: 4.08-4.36(\mathrm{~m}, 4 \mathrm{H}) \\
\mathrm{CH}_{3}: 1.33-1.40(\mathrm{~m}, 6 \mathrm{H})\end{array}$ & -- & $-1.28,7.97$ \\
\hline $3 c$ & $\begin{array}{c}\mathrm{P}-\mathrm{CH}_{2}: 4.25(\mathrm{~d}, J=7.14 \mathrm{~Hz}, 2 \mathrm{H}) \\
\mathrm{OCH}_{2}: 3.71-4.28(\mathrm{~m}, 4 \mathrm{H}) \\
\mathrm{CH}_{3}: 1.22-1.36(\mathrm{~m}, 6 \mathrm{H}) \\
\mathrm{COOH}: 9.32(\mathrm{~s}, 1 \mathrm{H})\end{array}$ & -- & $-2.08,6.93$ \\
\hline $3 d$ & $\begin{array}{l}\mathrm{Ar}-\mathrm{H}: 7.16-7.89(\mathrm{~m}, 4 \mathrm{H}) \\
\mathrm{OCH}_{2}: 3.88-4.32(\mathrm{~m}, 4 \mathrm{H}) \\
\mathrm{CH}_{3}: 1.24-1.38(\mathrm{~m}, 6 \mathrm{H})\end{array}$ & -- & -1.29 \\
\hline $3 e$ & $\begin{array}{c}\mathrm{Ar}-\mathrm{H}: 7.26-8.03(\mathrm{~m}, 4 \mathrm{H}) \\
\mathrm{OCH}_{2}: 3.60-4.21(\mathrm{~m}, 4 \mathrm{H}) \\
\mathrm{CH}_{3}: 1.22-1.42(\mathrm{~m}, 6 \mathrm{H})\end{array}$ & -- & $-1.72,10.64$ \\
\hline $3 f$ & $\begin{array}{c}\mathrm{Ar}-\mathrm{H}: 7.17-8.33(\mathrm{~m}, 4 \mathrm{H}) \\
\mathrm{OCH}_{2}: 4.09-4.15(\mathrm{~m}, 4 \mathrm{H}) \\
\mathrm{CH}_{3}: 1.32-1.37(\mathrm{~m}, 6 \mathrm{H})\end{array}$ & -- & -1.29 \\
\hline $3 g$ & $\begin{array}{c}\mathrm{Ar}-\mathrm{H}: 7.29-7.83(\mathrm{~m}, 4 \mathrm{H}) \\
\mathrm{OCH}_{2}: 4.01-4.13(\mathrm{~m}, 4 \mathrm{H}) \\
\mathrm{CH}_{3}: 1.21-1.36(\mathrm{~m}, 6 \mathrm{H}) \\
\mathrm{CHO}: 9.96(\mathrm{~s}, 1 \mathrm{H})\end{array}$ & $\begin{array}{c}190.64,135.69,133.21,128.25 \\
(\mathrm{~d}, J=4.7 \mathrm{~Hz}), 127.86,62.83 \\
\quad(\mathrm{~d}, J=7.1 \mathrm{~Hz}), 15.95\end{array}$ & $-1.50,20.71$ \\
\hline $3 \mathbf{h}$ & $\begin{array}{c}\mathrm{Ar}-\mathrm{H}: 7.21-7.35(\mathrm{~m}, 4 \mathrm{H}) \\
\mathrm{OCH}_{2}: 3.62-4.09(\mathrm{~m}, 4 \mathrm{H}) \\
\mathrm{CH}_{3}: 1.16-1.32(\mathrm{~m}, 6 \mathrm{H}) \\
\mathrm{CH}_{2}: 4.57(\mathrm{~s}, 2 \mathrm{H})\end{array}$ & -- & $-1.66,5.74$ \\
\hline $3 \mathbf{i}$ & $\begin{array}{c}\mathrm{Ar}-\mathrm{H}: 7.67(\mathrm{~d}, J=7.1 \mathrm{~Hz}, 2 \mathrm{H}) \\
7.35(\mathrm{~d}, J=7.3 \mathrm{~Hz}, 2 \mathrm{H}) \\
\mathrm{OCH}_{2}: 4.08-4.19(\mathrm{~m}, 4 \mathrm{H}) \\
\mathrm{CH}_{3}: 1.21-1.39(\mathrm{~m}, 6 \mathrm{H}) \\
\mathrm{OCH}_{3}: 3.77(\mathrm{~s}, 3 \mathrm{H})\end{array}$ & $\begin{array}{c}154.63,128.16,118.04,114.28 \\
63.30(\mathrm{~d}, J=4.5 \mathrm{~Hz}), 57.32,15.66 \\
(\mathrm{~d}, J=5.7 \mathrm{~Hz})\end{array}$ & $-1.38,7.04$ \\
\hline $3 \mathbf{j}$ & $\begin{array}{c}\mathrm{CH}_{2}: 4.31(\mathrm{~s}, 4 \mathrm{H}) \\
\mathrm{OH}: 4.82(\mathrm{brs}, 2 \mathrm{H}) \\
\mathrm{OCH}_{2}: 4.08-4.19(\mathrm{~m}, 4 \mathrm{H}) \\
\mathrm{CH}_{3}: 1.33-1.37(\mathrm{~m}, 6 \mathrm{H})\end{array}$ & $\begin{array}{c}66.22,63.51(\mathrm{~d}, J=4.1 \mathrm{~Hz}), 48.18 \\
15.81(\mathrm{~d}, J=5.4 \mathrm{~Hz})\end{array}$ & -1.50 \\
\hline
\end{tabular}



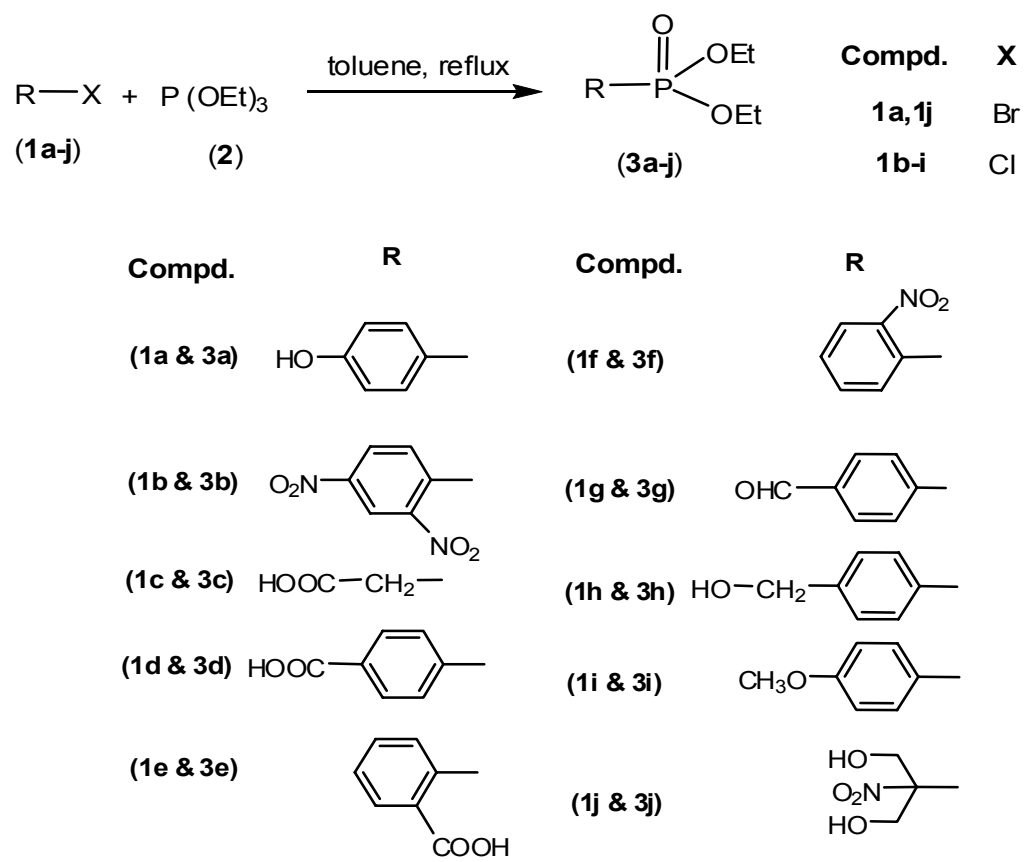

\section{Scheme 1}

\section{Antibacterial activity}

The compounds were diluted in DMSO for bioassay. Solvent control was included although no antibacterial activity has been noted for the solvent employed. Ciprofloxacin (Hi-media) controls were included to compare with compounds 3a-j. All samples were tested in triplicate and average results were recorded.

The compounds were assayed for antibacterial activity against six registered bacterial isolates which were obtained from the NCIM (National Collection of Industrial Microorganisms, National Chemical Laboratories, Pune-411 003, India). The bacteria included Gram positive bacterial isolates-Staphylococcus aureus (NCIM No: 5021, ATCC No. 25923), Bacillus faecalis (NCIM No: 2063, ATCC No. 6633) and four Gram negative bacteria-Escherichia coli (NCIM No: 2931, ATCC No. 25922), Pseudomonas aeruginosa (NCIM No: 5029, ATCC No: 27853), Salmonella typhimurium (NCIM No: 2501, ATCC No: 23564) and Klebsiella pneumoniae (NCIM No: 2957). The bacteria were grown on Hi-media nutrient agar and sub cultured as needed. 


\section{Experimental Section}

General Procedures. All chemicals were commercial products and distilled / recrystallised before use. Elemental analyses were performed by the Central Drug Research institute, Lucknow, India. IR spectra were recorded as $\mathrm{KBr}$ pellets and Nujol mulls on a Perkin Elmer 283 unit. The ${ }^{1} \mathrm{H},{ }^{13} \mathrm{C}$ and ${ }^{31} \mathrm{P}$ NMR spectra were taken on AMX $400 \mathrm{MHz}$ spectrometer operating at $400 \mathrm{MHz}$ for ${ }^{1} \mathrm{H}, 100$ $\mathrm{MHz}$ for ${ }^{13} \mathrm{C}$ and $161.9 \mathrm{MHz}$ for ${ }^{31} \mathrm{P}$. All these compounds were dissolved in DMSO- $d_{6}$. The chemical shifts in $\delta$ were referenced to TMS $\left({ }^{1} \mathrm{H}\right.$ and $\left.{ }^{13} \mathrm{C}\right)$ and $85 \% \mathrm{H}_{3} \mathrm{PO}_{4}\left({ }^{31} \mathrm{P}\right)$.

General procedure for products 3a-j. In a flame-dried three-necked flask the appropriate aryl / alkyl halide $(0.001 \mathrm{~mol})$ was mixed with triethyl phosphite $(0.249 \mathrm{~g}, 0.0015 \mathrm{~mol})$ and stirred at reflux temperature for 6-8 hrs and protected with a $\mathrm{CaCl}_{2}$-tube, respectively. After the completion of reaction (monitored by TLC), the oily product was obtained. The product was purified by column chromatography on silica gel using petroleum ether-ethylacetate (7:3) as eluent.

Table 3. Antibacterial activity of compounds 3a-j in terms of DIZ in mm

\begin{tabular}{|c|c|c|c|c|c|c|c|c|c|c|c|c|}
\hline \multirow{3}{*}{ Compd. } & \multicolumn{2}{|c|}{$\begin{array}{c}\text { Staphylococcus } \\
\text { aureus }\end{array}$} & \multicolumn{2}{|c|}{$\begin{array}{l}\text { Bacillus } \\
\text { faecalis }\end{array}$} & \multicolumn{2}{|c|}{$\begin{array}{c}\text { Escherichia } \\
\text { coli }\end{array}$} & \multicolumn{2}{|c|}{$\begin{array}{c}\text { Pseudomonas } \\
\text { aeruginosa }\end{array}$} & \multicolumn{2}{|c|}{$\begin{array}{l}\text { Salmonella } \\
\text { typhimurium }\end{array}$} & \multicolumn{2}{|c|}{$\begin{array}{c}\text { Klebsiella } \\
\text { pneumoniae }\end{array}$} \\
\hline & $\vec{\Xi}$ & $\vec{\Xi}$ & $\vec{\Xi}$ & $\vec{\Xi}$ & 弱 & $\vec{\Xi}$ & $\vec{\Xi}$ & $\vec{\Xi}$ & $\vec{\Xi}$ & $\vec{\Xi}$ & $\vec{\Xi}$ & $\vec{\Xi}$ \\
\hline & 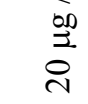 & $\begin{array}{l}\frac{50}{2} \\
\frac{9}{2}\end{array}$ & $\frac{\text { so }}{3}$ & $\begin{array}{l}\frac{50}{3} \\
\frac{q}{q}\end{array}$ & 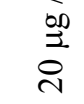 & $\begin{array}{l}\frac{50}{3} \\
\frac{q}{q}\end{array}$ & 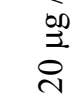 & $\frac{\frac{50}{2}}{9}$ & 势 & $\frac{\text { on }}{2}$ & $\frac{\text { on }}{2}$ & $\begin{array}{l}\frac{50}{3} \\
\frac{9}{2}\end{array}$ \\
\hline $3 a$ & + & ++ & - & + & + & ++ & + & ++ & - & + & + & ++ \\
\hline $3 b$ & + & ++ & - & + & - & + & - & + & - & + & - & + \\
\hline $3 c$ & - & ++ & - & + & - & ++ & + & ++ & - & + & + & ++ \\
\hline 3d & + & ++ & - & + & - & + & - & + & - & + & - & + \\
\hline $3 e$ & + & ++ & + & ++ & + & ++ & + & ++ & + & ++ & + & ++ \\
\hline $3 f$ & - & + & - & + & - & + & - & + & + & ++ & - & + \\
\hline $3 g$ & - & + & - & + & - & + & - & + & - & + & - & + \\
\hline $3 \mathrm{~h}$ & + & ++ & + & ++ & + & ++ & + & ++ & - & + & + & ++ \\
\hline $3 \mathbf{i}$ & + & ++ & - & + & - & + & - & + & - & + & - & + \\
\hline $3 \mathbf{j}$ & + & ++ & - & + & - & + & - & + & - & + & - & + \\
\hline Cifrofloxacin & \multicolumn{2}{|c|}{22} & \multicolumn{2}{|c|}{24} & \multicolumn{2}{|c|}{30} & \multicolumn{2}{|c|}{25} & \multicolumn{2}{|c|}{2} & \multicolumn{2}{|c|}{25} \\
\hline
\end{tabular}

'+' indicates 10-12 mm. '++' indicates $12-15 \mathrm{~mm}$. '-' indicates no activity.

Disc diffusion bioassay. For bioassay suspension of approximately $1.5 \times 10^{8}$ bacterial cells per $\mathrm{mL}$ were used. In sterile normal saline was prepared as described by Forbes et $a l^{17}$ and $1.5 \mathrm{~mL}$ of it was uniformly spread on Nutrient Agar (Hi-media) in 12 x $1.2 \mathrm{~cm}$ glass Petri dishes, left aside for $15 \mathrm{~min}$ and excess of suspension was then drained and discarded properly. For the 
agar disc diffusion method, the test compound was introduced onto the disc and then allowed to dry. Thus the disc was completely saturated with the test compound. Then the disc was introduced onto the upper layer of the medium with the bacteria. The petri dishes were incubated overnight at $37^{\circ} \mathrm{C}$ for $24 \mathrm{hrs}$. Bioactivity was determined by measuring Diameter of Inhibition Zones (DIZ) in mm. The compounds' 3a-j concentrations were taken as 20 and $40 \mu \mathrm{g} / \mathrm{mL}$ were evaluated for disc method. Each test was done in triplicate and the mean of the diameter of the inhibition zones was calculated. Controls included the use of solvent without test compounds although no antibacterial activity was noted for the solvent employed in the test ${ }^{18}$ (Table 3).

Determination of minimum inhibitory concentration (MIC). Minimum inhibitory concentration (MIC) was determined for the compounds $\mathbf{3 a - j}$. The concentration at which there was no visually detectable bacterial growth was taken as MIC. Compounds 3a-j concentrations of $0.1-5.6 \mathrm{mg} / \mathrm{mL}$ in steps of $100 \mu \mathrm{g} / \mathrm{mL}$ were evaluated. Specifically $0.1 \mathrm{~mL}$ of standardized inoculum $\left(1-2 \times 10^{7} \mathrm{CFU} / \mathrm{mL}\right)$ was added to each tube. The tubes were incubated aerobically at $37{ }^{\circ} \mathrm{C}$ for $18-24 \mathrm{hrs}$. Two control tubes were maintained for each test batch. These included antibiotic control (tube containing compounds $\mathbf{3 a - j}$ and the growth medium without inoculum) and organism control (the tube containing the growth medium, physiological saline and the inoculum). The lowest concentration (highest dilution) of the compounds $\mathbf{3 a - j}$ that produced no visible bacterial growth (no turbidity) when compared with the control tubes was regarded as $\mathrm{MIC}^{18}$ (Table 4).

Table 4. Minimum inhibitory concentration (MIC) $\mathrm{mg} / \mathrm{mL}$

\begin{tabular}{ccccccc}
\hline Compd. & $\begin{array}{c}\text { Staphylococcus } \\
\text { aureus }\end{array}$ & $\begin{array}{c}\text { Bacillus } \\
\text { faecalis }\end{array}$ & $\begin{array}{c}\text { Escherichia } \\
\text { coli }\end{array}$ & $\begin{array}{c}\text { Pseudomonas } \\
\text { aeruginosa }\end{array}$ & $\begin{array}{c}\text { Salmonella } \\
\text { typhimurium }\end{array}$ & $\begin{array}{c}\text { Klebsiella } \\
\text { pneumoniae }\end{array}$ \\
\hline 3a & 3.6 & 4.0 & 3.3 & 3.6 & 4.0 & 3.8 \\
3b & 3.8 & 4.1 & 4.0 & 4.1 & 4.2 & 4.0 \\
3c & 4.0 & 4.2 & 3.9 & 3.8 & 4.4 & 3.7 \\
3d & 5.4 & 5.3 & 4.2 & 4.6 & 4.0 & 4.3 \\
3e & 2.8 & 2.6 & 2.7 & 3.0 & 2.8 & 3.2 \\
3f & 4.4 & 4.3 & 4.2 & 4.6 & 3.9 & 4.0 \\
3g & 5.2 & 5.0 & 4.9 & 4.8 & 5.4 & 4.2 \\
3h & 3.6 & 3.2 & 3.8 & 3.4 & 4.4 & 3.9 \\
3i & 3.0 & 5.0 & 4.0 & 4.4 & 5.6 & 4.2 \\
3j & 3.7 & 3.9 & 4.2 & 4.4 & 4.0 & 4.1 \\
\hline
\end{tabular}

\section{Conclusions}

We synthesized bioactive and novel phosphonates 3a-j in high yield by Michaelis-Arbuzov reaction without any catalyst. They showed moderate antibacterial activity against selected bacteria. Among all these compounds $\mathbf{3 e}$ showed highest antibacterial activity at lower 
concentration against both Gram negative and Gram positive bacteria. Compounds 3g, 3i showed lowest activity even at highest concentrations. Compound 3d showed highest activity against Gram positive bacteria when compared with Gram negative bacteria.

\section{Acknowledgements}

The authors express thanks to Prof. C. Devendranath Reddy and Dr. C. Naga Raju, Dept. of Chemistry, S. V. University, Tirupati, for helpful discussions and SIF, IISc, Bangalore, for providing the NMR spectra.

\section{References}

1. Sikorski, J. A.; Logusch, E. W. Aliphatic carbon-phosphorus compounds as herbicides. In Handbook of Organophosphorus Chemistry; Engel, R. Ed.; Marcel Dekker: New York, 1992; pp739.

2. Eto, M. Phosphorus containing insecticides. In Handbook of Organosphorus Chemistry; Engel, R. Ed.; Marcel Dekker; New York, 1992; pp 807.

3. Engel, R. Chem. Rev. 1977, 77, 349.

4. Miller, P. S. Non-ionic antisense oligonucleotides. In Oligodeoxynucleotides-Antisense Inhibitors of Gene Expression; Cohen, J. S. Ed.; Macmillan: New York, 1989; pp79.

5. (a) Michaelis, A.; Kaehene, R. Chem. Ber. 1898, 31, 1408. (b) Arbuzov, A. E. J. Russ. Phys. Chem. Soc. 1906, 38, 687.

6. (a) Kosolapov, G. Organophosphorus Compounds; Wiley: New York, 1950; Chapter 7. (b) Harvey, R. G.; DeSombre, E. R. Topics in Phosphorus Chemistry 1964, Vol. I, Grayson, M.; Griffith, E. J.; Ed.; Interscience: New York, p 57. (c) Arbuzov, B. A. Pure Appl. Chem. 1964, 9, 307. (d) Henning, H. G.; Hilgetag, G. Z. Chem. 1967, 7, 169 (e) Bhattacharya, A. K.; Thyagarajan, G. Chem. Rev. 1981, 81, 415.

7. Sardarian, A. R.; Kaboudin, B. Synth. Commun. 1997, 27, 543.

8. Sardarian, A. R.; Kaboudin, B. Tetrahedron Lett. 1997, 38, 2543.

9. Kaboudin, B. J. Chem. Research (s) 1999, 402.

10. Balthazor, T. M.; Grabiak, R. C. J. Org. Chem. 1980, 45, 5425.

11. Tavs, P.; Korte, F. Tetrahedron 1967, 23, 4677.

12. Lu, X.; Zhu, J. Synthesis 1987, 726.

13. Erker, T.; Handler, N. Synthesis 2004, 668.

14. Thomas, L. C. The Interpretation of the Infrared Spectra of Organophosphorus Compounds, Heydon \& Sons Ltd., London, 1974.

15. Silverstein, R. M.; Bassler, G. C.; Morill, T. C. Spectrometric Identification of Organic Compounds, $6^{\text {th }}$ Edn., John Wiley \& Sons: New York, 1991. 
16. (a) Quin, L. D.; Verkade, J. G. Phosphorus-31 NMR Spectral Properties in Compound Characterization and Structural Analysis, VCH: New York, 1994. (b) Haranath, P.; Anasuyamma, U.; Syam Prasad, G.; Naga Raju, C.; Suresh Reddy, C. Heterocyclic Commun. 2004, 10, 457. (c) Haranath, P.; Sreedhar Kumar, V.; Suresh Reddy, C.; Naga Raju, C.; Devendranath Reddy, C. J. Heterocyclic Chem. 2007, 44, 1.

17. Forbes, B. A.; Sahm O. F.; Weissfeld, A. S.; Trevomp, E. A., Methods for Testing Antimicrobial Effectiveness. In: Bailey and Scott's Diagnostic Microbiology MOS by Co: St Louis, Missouri, 1990, 171.

18. Shahidi Bonjar, G. H. Asian J. Plant Sci. 2004, 3, 56. 